\title{
A Review of Telepsychology and Mental Health Mobile Apps in Advanced Countries: Opportunities for Ghana's Mental Health Care
}

\author{
Evelyn 0wusu* \\ Regent University College of Science and Technology, Ghana
}

*Corresponding author: Evelyn Owusu, Regent University College of Science and Technology, Accra-Ghana

\begin{abstract}
Sustainable development denotes the development of nations in ways that are not restrictive for future generations in their efforts to meet their own needs. Without healthy human beings, sustainable development cannot be successfully carried out or assessed as a goal. For this reason, health, which forms the third arm of sustainable development goals have received much attention across nations. In Ghana, however, the aspect of health which has not received as much attention lies in the area of mental health. This paper addresses how mental health care and access in Ghana can be improved through the use of applied science and technology. Specifically, recommendations are made about the prospects that telepsychology and mental health mobile apps hold for applications in Ghana. This concept is introduced in the paper by reviewing telemental health practices and the use of mobile mental health apps in more advanced countries like the United States and Britain. The paper addresses how these two concepts can be introduced in Ghana, and guided by the experiences of other African countries, the potential associated challenges of implementation are also discussed. The paper ends with recommendations on how to overcome these challenges and makes note of the impossibility of fully achieving sustainable development goals for health when Ghana's mental health care system remains in such dire states.
\end{abstract}

Keywords: Sustainable development; Ghana; Mental health; Telepsychology; Mental health mobile apps

\section{Introduction}

In September 2015, 194 countries adopted the sustainable development goals spearheaded by the United Nations. These 17 goals officially came into effect in January 2016 with the ultimate aim of a higher quality of life for all ("The Sustainable Development Agenda," n.d.). Sustainable development can be defined as development that meets the needs of the present without compromising the ability of future generations to meet their own needs. Sustainable development implies development and growth in conscious ways that do not cause havoc for future generations. Human beings are the central reason for the need for sustainable development and at the same time, without humans, the agenda for sustainable development cannot be realized. Health and wellbeing comprise the third arm of the United Nations' sustainable development goals.

The goals of sustainable development cannot be achieved when there is a high prevalence of encumbering illnesses (Von
Schirnding \& Mulholland [1]). Health is a significant contributor to, and an indicator of, sustainable development and its wide-reaching impact on people, societies, countries, economies and such goals as the sustainable development goals have corralled attention to this integral area. In doing so, there is an aspect of health that has not gained as much attention as the physical health related issues in Ghana and this lies in the area of mental health. Mental health can be defined as a state of well-being in which every individual realizes his or her own potential, can cope with the normal stresses of life, can work productively and fruitfully, and is able to make a contribution to his or her community ("WHO | Mental health: a state of well-being", 2017). It comprises an individual's psychological, emotional, and social being with impacts on the way one thinks, feels and acts. The state of an individual's mental health affects how stress is handled, how relationships are managed, and the decisions that one makes. Just like physical health, mental health is important in every phase of life, from childhood to adulthood. In childhood for 
example, mental health plays a role in the bond between parents and their children. It helps set a solid foundation for life. During adolescence, mental health plays an integral role in the way the adolescent develops a sense of morality, and in adulthood, mental health plays a significant role in the way work, family and intimate relationships develop and evolve. In a pandemic such is currently being experienced with the coronavirus, its associated distresses of grief, social isolation, fear, panic and pre-existing mental health conditions mandate the pursuit of mental health can be ameliorative.

Problems with mental health can come in mild, moderate or severe forms. They can impact how one deals with mandatory social isolation or social distancing during a pandemic. They can impact a child's understanding of social cues, for example, as can be the case with Autism Spectrum Disorders. Issues with mental health can influence the way the student is able to take in, process and understand information as can be the case with learning disorders. In the case of the new mother, problems with mental health can impact the way she is able take care of her child as can be the case with post-partum depression. Early detection and intervention tend to yield better outcomes for individuals with mental health disorders. In Ghana, however, access to mental health care and mental health professionals are severely lacking. According to the World Health Organization's situational analysis report on Ghana, there is a mental health treatment gap of $98 \%$ in Ghana, meaning that only 2 out of every 100 Ghanaians with mental health issues will get the care they need. Advancements in technology have played a crucial role in the evolution of health care treatment and prevention. From electronic medical record keeping systems to portable diagnostic devices, health care systems are making use of technological advancements that bring about more efficiency. Diagnostic devices are being produced to be more sensitive in ways that can lead to early diagnosis and now, because of technology, healthcare devices are being made in sophisticated ways that allow for transfer and use in countries and areas that do not have easy access to such mechanisms. The impact that technology has had on medical science has been enormous and continues to evolve daily. In the field of psychology, technology has had impacts although not as great as that of the field of medicine. During a pandemic such as the world is currently facing with the coronavirus, many occupational fields, not just medical or psychological are turning to technology to provide care and services. Ghana can explore ways that technology can positively influence mental health care and access. This paper explores a few of these ways.

\section{Methodology}

The methodology for this research paper involved a review of the telepsychology practices and mobile mental health app use in advanced countries, particularly the United States and the United Kingdom. The APA and BPS telepsychology guidelines were reviewed in addition to the United Kingdom's National
Health Service digital library for mobile mental health apps and the APA's mobile mental health app evaluation model. These countries were selected based on the major advances they have made with regard to regulating the use of telepsychology practice and mobile mental health apps. A literature review was also conducted on the application of telepsychology and mental health mobile apps in other African countries. This review was conducted mainly to balance the opportunities of telepsychology and mobile mental health apps observed in the advanced countries like the United States and United Kingdom with the potential real-life implementation challenges that have been discovered in similar cultural contexts.

\section{Review of Telepsychology and Mental Health Mobile Apps}

The trends of telepsychology and mental health mobile apps in more advanced countries can serve as learning opportunities for Ghana's mental health system. The following sub-sections discusses some of these opportunities and makes suggestions for implementation [2-4].

\section{Telepsychology and mental health care access}

Telepsychology can be defined as the provision of psychological services using telecommunication technologies. Psychological services provided with such a modality can be in lieu of or in addition to traditional therapy or treatment. With telecommunication mechanisms like e-mail, texting, mobile apps and videoconferencing, mental health services can be provided without the need for inperson interactions. Telepsychology presents as a unique option for patients who are not ambulatory or for people and families who are unable to travel to the psychologist's office to access services. For individuals whose diagnoses (such as agoraphobia or the fear of social situations) prevent them from leaving the house to get to the psychologist, telepsychology presents as an opportunity to begin the process of treatment that would eventually help them overcome their fear and potentially transfer to in-person treatment. The practice of some form of telepsychology or online counseling is not a novel concept in Ghana or abroad. Telepsychology has been around since the launch of the internet in 1972 (Alleman [2]). Currently, psychologists worldwide perform or have performed some sort of online counseling or telepsychology. In 2008, a survey conducted by the American Psychological Association revealed that 87 percent of psychologists provide mental health care services via telepsychology (American Psychological Association, 2009). The prevalence of this practice has led to the formulation of practice guidelines in some developed countries like the United States, Britain, and Canada. Mental health care access is an area in Ghana's mental health care system that requires major development. With the exception of the popular, major psychiatric hospitals in the country, it can be difficult for the average Ghanaian to know where to go to in order to seek mental health care especially one that is 
not located in a psychiatric hospital. In recent years, the Ministry of Health has made provisions to allow for the placement of clinical psychologists in its hospitals. However, mental health care access continues to be a problem because of location, insufficient numbers of clinical psychologists and stigma among other reasons. Today, one is more likely to gain access to a clinical psychologist in the nation's capital of Greater Accra than in any of the other regions of Ghana. This problem is worsened by reported estimates from Osei [3], the former Chief Psychiatrist of the Accra Psychiatric Hospital of a doctor-patient ratio of 1:1.7 million in the mental health sector (Awaf [4]). Stigma can be a deterrent when it comes to seeking mental health care even among people who have relatively easy access to these services. With clinical psychology facilities sometimes being housed in places like the Accra Psychiatric Hospital, individuals may be afraid to seek out these services for fear of being perceived as 'mad'. In cases like these, the practice of telepsychology holds the potential for ameliorating such problems by abbreviating the distance between the prospective patient and psychologist, for allowing easy access to psychologists and for reducing the potential stigma of accessing the psychologist in a setting that could potentially be stigmatizing.

\section{Development of telepsychology guidelines and opportunities for ghana}

In 2013, the American Psychological Association (APA) released their guidelines for the practice of telepsychology. Prior to that in September 2009, the British Psychological Society (BPS) released its second edition of regulations concerning the provision of psychological services via the internet and other nondirect means (Professional Practice Board [5]). The formation of these guidelines was a response to the increasing rate of the use of telecommunication strategies among its psychologists. While the APA and BPS recognized the potential for telepsychology to increase access to psychological services, it also recognized the need for the formal regulation of tele psychological practices because of the potential challenges it presented. It is possible for Ghana to take a cue from these guidelines in order to pre-empt any potential challenges, and to keep itself from being reactive when tele psychological services rise to the capacities experienced in more developed countries. The formulation of such a guideline for Ghana's psychologists will also serve as a mechanism to permit the use of such a service delivery mechanism as a solution to Ghana's mental health care access problems [6]. For the APA (and BPS), core issues identified in their guidelines for telepsychology include the competence of the psychologist in providing tele psychological services and the importance of the need for the psychologist to be well-versed in the use of the telecommunication technologies as well as the awareness of the potential impact of such a modality on clients/patients, supervisees and other relevant stakeholders ("Guidelines for the Practice of Telepsychology", 2013). There is a vast array of choices when it comes to telecommunication technologies today and it is important for the psychologist using such modalities to be knowledgeable about their chosen systems of communication especially where issues of privacy and confidentiality are concerned. Given the sensitivity of information that is shared in the client-psychologist relationship, the potential fears of stigma as well as the consequences of client-psychologist privileged information becoming public knowledge, it is important for the psychologist to be thoroughly knowledgeable about their telecommunication mechanisms and its privacy/confidentiality limitations in order to select systems that provide the most privacy/ confidentiality possible and to communicate any such limitations to the patient/client as sanctioned by psychological practice.

The upholding of the standards of care in the delivery of telepsychology services was also an important issue in the development of the APA and BPS telepsychology guidelines. By these, the guidelines reinforced the importance of translating the same ethical and professional practices of in-person services to telepsychology. While telecommunication technologies have the potential of connecting the patient to the psychologist, it also has the potential of creating a disconnect in the patient-psychologist therapeutic relationship. The patient/client who 'sees' the psychologist via a telecommunication device may not feel as close to the psychologist as the patient/client who does so face-to-face or in-person. In the patient- psychologist relationship, trust and the feeling of safety are important, and these tend to be more easily developed through in-person interactions.

For this reason, the APA and BPS telepsychology guidelines advise that psychologists choose telecommunication systems that do not negatively impact the development of a good patientpsychologist therapeutic relationship. In the spirit of upholding the ethical and professional standards of practice, it is also important that the psychologist assesses the effectiveness of telepsychology modalities to meet the needs of the client. While mental health care access can be a problem, telepsychology should be an option which not only connects the patient to the psychologist but which also does so in a way that ensures the patient is receiving optimal ethical and professional standards of care. Ethical and professional standards of care cannot be sacrificed on the altar of mental health care access. Sometimes, the severity of a mental health issue is such that an in-person service is required, and treatment cannot ethically be provided via the practice of telepsychology.

An area of focus that the APA guidelines concentrated on and which might potentially require some significant adaptation for Ghana as compared to the other identified core areas lies in the area of psychological testing. Psychological testing is equivalent to the laboratory testing that is performed in the medical sciences in that it is also needed for aiding in diagnoses formation and the assessment of the current functioning of the patient. While there are online psychological testing options, most tests are designed for in- person interactions allowing for the observed behaviors of 
patients/clients during the testing process to serve as important information in the testing process. For example, if a cognitive test is being conducted on a child who ends up scoring above average, it is also important to note if the child was attentive during the testing process or if the child had difficulty staying seated. It is important to note if the child was able to follow social cues or the child answered questions while staring out a window without making eye contact with the psychologist. While testing via videoconferencing can provide such information, it can be difficult to ascertain what part the videoconferencing in itself contributes to the behaviors witnessed in the patient/client during the testing process. Psychological testing in Ghana already possesses issues given the fact that the available testing tools are designed for a foreign population. This can create issues regarding validity and reliability of tests results for the Ghanaian cultural context without the additional variable of a telecommunication system of testing. Still, with the goal of the maintenance of ethical and professional standards of practice, guidelines can be formulated that ensures integrity of practice and the provision of appropriate care while trying to solve the problem of mental health care access.

\section{Mobile apps and mental health care access}

Mobile applications serve as mechanisms through which the problem of mental health care access can also be solved or improved upon. A mobile application or mobile app is an application software that is designed to run on mobile devices such as smart phones or tablets. With advances in technology, it is more common now to find individuals using smart phones which come with the capability of having apps such as those for emails, social media, messaging, shopping, gaming, and other areas of interest. Today, some smart phones have already built-in mobile apps that are geared towards health. Some apps for example allow people to track their food and water intake, physical activity and even stress levels. There are mental health apps (also known as mHealth apps) too that, for example, provide support for teenagers struggling with depression like the Code Blue app and apps that guide people through breathing exercises for stress management like the Breathe2Relax app. Some of these mental health apps are also interactive, combining therapeutic techniques with real life experts like the Lantern app. Other apps that can be used in addition to real life treatment include mobile applications such as the PTSD Coach, Self-Help for Anxiety Management (SAM) and Optimism apps which allow people to track their symptoms over time while providing psychological tools ("Top 10 Mental Health Apps").

As already discussed, the problem of mental health care access in Ghana is impacted by the lack of clinical psychologists in the country, the physical distance between the patient and psychologist as well as the inability of people to know where and how to contact a psychologist, especially one that is not located in a psychiatric hospital setting. While accessing mental health services in a psychiatric hospital setting is not a problem in itself, there can be a hesitancy to access services in such locations because of the potential stigma of being perceived as 'mad.' Mobile applications have been used as a bridge to mental health care access problems in countries like the United States and Britain. mHealth apps can be designed where people in Ghana have a way of accessing available clinical psychologists and facilities by location and specialty. These apps can have built in the ability of also getting in touch with or communicating with a psychologist to begin treatment. Such an app also has the potential of regulating professional psychology practice in the country if the app only includes psychologists and facilities in the country which are actively licensed. When a psychologist or facility ceases to maintain active licensure, measures can be put in place to withdraw inclusion in the app or deactivate visibility and access to such psychologists and facilities. With such an app approved by the Ghana Psychological Council, psychologists will want to ensure active licensure of themselves and their facilities while potential patients are assured of getting access to qualified and approved professionals and facilities. Such an app which provides information on the psychologist, location and specialty will also serve as important data for developing the profession in the country. For example, if the app indicates the need for more psychologists who work with geriatric populations, geriatric training programs can be developed to cater for the mental health needs of such a population in the country. In mental health treatment, clinicians sometimes make use of group therapy to help support individuals who struggle through the same symptoms or problems. For example, group therapy can be used with individuals struggling with substance use, individuals who have gone through grief and loss, and individuals who struggle with depression and anxiety. This group treatment modality allows individuals to receive support from others who experience the same problems they go through and whom can empathize with their struggle. It also presents with opportunities for these individuals to hold each other accountable to treatment goals and to have a sense of purpose through their ability to contribute to the lives of others like themselves who are hurting thus raising self-esteem. There are apps that Ghana can adapt or develop that provide the platform to offer group therapy with a trained therapist. Such an app can be helpful for communal support through mental health struggles without some of the potential reservation and risks to confidentiality that the typical face-to-face group therapy presents.

In the traditional face-to-face group therapy setting, there are times when an individual or groups of individuals may not be as open or as vocal in ways that allow them to contribute to and benefit from group treatment. When the option of such an app is available, this can allow those who would likely be more hesitant and withdrawn to be more vocal and open. In addition to the group therapy module, mental health mobile apps present as a good opportunity for complementing in-person treatment or the practice 
of telepsychology. mHealth apps can provide supportive services to patients and clients between sessions that help facilitate treatment. The individual who is seeing a psychologist for anxiety related problems, for example, can have an app that provides resources for coping such as breathing exercises.

According to Henderson et al. [7] , one factor that affects the seeking out of mental health services includes lack of knowledge to identify features of mental illnesses. It is possible for people with mental health problems to experience difficulties that they either do not understand or which they brush aside. Sometimes the responsibilities of daily life can prevent one from attending to mental health problems until symptoms reach a point where they become severe enough to require psychiatric hospitalization or attempts are made to commit suicide. There is a great need for Ghanaians to be educated on mental health and through psychoeducation, convey the importance of seeking help while normalizing mental health help seeking behaviours to that of physical health seeking behaviors. When people are made to conceptualize seeking mental health help the same way they would seek medical help, fears associated with mental health stigma can be reduced and we could possibly see a decline in suicide behaviors. Mental health apps that are geared towards psychoeducation can be useful in Ghana's fight for mental health education, access, and care. Such mental health apps can include screening for mental health issues which would have the potential for making on the spot referral recommendation when applicable. For example, an individual experiencing symptom of depression can take a questionnaire on the mHealth app that indicates their level of depression (whether mild, moderate, or severe) and makes recommendations to see a psychologist.

\section{Development of mhealth apps and opportunities for ghana}

The American Psychological Association has developed an app evaluation model in response to the increasing use of mHealth technologies. This decision to develop the evaluation model was also a response to questions received from mental health care professionals concerning the efficacy and risks of these mobile mental health apps. It appears the expanding use of mobile mental health apps was such that the development of the evaluation model was a better alternative to running every available app through an approval process and advertising those as APA-approved. In contrast to the stance taken by the American Psychological Association, the United Kingdom's National Health Service has included in its digital library, a list of mhealth apps with indications of which apps have been approved and which apps are in the process of being tested by NHS. There are legitimate concerns regarding the increasing number of mHealth apps and the associated tendency for people to self-diagnose or use such mechanisms in lieu of the necessary professional care. The increased access to the internet in general has come with the tendency for people to look up the symptoms they experience -whether physical or behavioral- in order to figure out what is wrong with them. The enormous and varied number of mHealth apps only provides more avenues for potential selfdiagnoses and misuse.

It is for this reason that Ghana's Psychological Council could combine the strategies used by both the APA and UK's NHS to formulate an evaluation model that is used to recommend apps for the public. With such a mechanism in place, mHealth apps can be reviewed and regulated by the appropriate professional national body from the very onset. Also, mHealth app developers can begin the app development process keeping in mind that the final product would have to go through an approval process before being used by the public. The Ghana Psychological Council or Association bodies can themselves develop approved apps for its professionals and countrymen and women as well. The apps that are locally developed would have to be suited to the Ghanaian context and take into consideration some of the cultural and unique expressions of mental health problems among Ghanaians, for example, the commonality of somatization of some mental health problems.

\section{Finding and Recommendation}

Telepsychology and mental health mobile apps hold unique opportunities for improving mental health care and access in Ghana when adopted. Like most opportunities, these two concepts present with challenges that will be discussed in the sections to follow with recommendations on how to overcome these obstacles in order to improve mental health care and access. When mental health care and access is improved upon, the nation's sustainable development goals for health can make gains towards a more complete picture of fulfillment. Ghana's sustainable development goals for health cannot be entirely met if its mental health care problems persist.

\section{Human health resources}

Telepsychology and mobile apps provide potential solutions to mental health care problems in Ghana, but certain issues currently impede its implementation. These issues are not unlike the issues discovered in an analysis of tele mental health in South Africa (Jefee-Bahloul et al., [8] ). Just like in South Africa, Ghana does not have sufficient human health resources. As already discussed, there aren't enough mental health professionals to meet the current demand in the nation. For this reason, there is the need for mental health or psychology training up to the highest level possible in the country. There is also the need for the profession to be properly supported by the government in order to keep from deterring other professional aspirants from the field of mental health care or psychology.

\section{Infrastructure}

An obvious potential limitation also lies in the requirements of technical and administrative support that the proposed technology 
mechanisms require. The use of telepsychology mandates adequate bandwidths that might not be available or which may be too expensive to acquire. Also, while messaging apps like WhatsApp and Skype can be used for telepsychology, these also require adequate amounts of data which could be relatively expensive when used at the levels that would be required for appropriate treatment. Again, should the problem of expense be addressed, another potential problem arises in the requirement of stable network connectivity which is not always the case in Ghana.

\section{Political will}

Until the year 2012 when Ghana's mental health bill was passed, the country operated under a mental health law that was put into effect in 1972. Although Ghana made tremendous strides with the passing of the 2012 mental health act, five years later, the Legislative Instruments which will serve to assist the implementation of the bill is yet to be passed (Boateng [5]). Improvement in mental health care and access through recommendations of modalities like telepsychology and the use of mental health mobile apps would also only be more successful with government or political backing. Without support from Ghana's government to improve upon mental health care, Ghana will likely continue to lag behind when it comes to attaining the advances that have been seen in the more developed countries with mental health care practice [9-11].

\section{Conclusion}

Sustainable development is development that meets the needs of the present without compromising the ability of future generations to meet their own needs. The health of human beings plays a central role in the fulfillment of sustainable development goals both as enactors and indicators of sustainable development. Health forms the third arm of the United Nation's sustainable development goals and has received great attention given its implications not just on sustainable development goals but to the lives of families, societies and economies. In meeting sustainable development goals for health, mental health has not received as much attention although it holds just as much significance in attaining sustainable development goals for health. Mental health can have just as significant an impact on families, societies and economies. An individual with a healthy body but an unhealthy mind can be just as incapable of functioning as an individual with a healthy mind but an unhealthy body. Telepsychology and mobile mental health apps present as great opportunities for mitigating mental health care access problems in Ghana. During and outside of pandemic realities, these technologies can serve as means of connecting the potential patient to the psychologist, discovering options for mental health care, regulating the practice of psychotherapy in the country, and providing psychoeducation and complementary resources to treatment. With continuing growth in Ghana's telecommunication technologies and support for the work of mental health professionals, the discussed challenges can be overcome and Ghana can make its way toward greater mental health care access, and ultimately, the achievement of its sustainable development goals for health.

\section{Conflict of Interest}

The author declares that there is no conflict of interest regarding the publication of this article. Disclaimer: This paper was presented at the ICAST 2019 Conference at the Kumasi Technical University in Ghana and published in the conference proceedings.

\section{References}

1. Vincent IO Agyapong, Akwasi Osei, Conor K Farren, Eilish McAuliffe (2015) Factors influencing the career choice and retention of community mental health workers in Ghana. Hum Resour Health 13:40-56.

2. Alleman J (2002) Online counseling: The Internet and mental health treatment. Psychotherapy: Theory, Research, Practice, Training 39(2):199-209.

3. Daniel Michalski, Tanya Mulvey, Jessica Kohout (2009) 2008 APA Survey of Psychology Health Service Providers. American Psychological Association Washington, DC pp:1-100.

4. Awaf A M (2016) Ghana news: Mental Health Care; Ghana among worst in Africa-Graphic Online. Graphic Online 8:3.

5. Boateng D (2017) Parliament urged to pass LI on Mental Health. Graphic Online 8:12.

6. American Psychologist (2017) Guidelines for the Practice of Telepsychology. American Psychological Association 68(9):791-800.

7. Henderson C, Evans Lacko S, Thornicrof G (2013) Mental Illness Stigma, Help Seeking, and Public Health Programs. American Journal of Public Health (AJPH) 103(5):777-780.

8. Jefee Bahloul H, Barkil Oteo A, Augusterfer E (2017) Telemental health in resource-limited global settings ( $1^{\text {st }}$ edn.), Oxford University Press, New York pp:51-66.

9. Michael Berger, Adrian Skinner (2009) The provision of psychological services via the internet and other non-direct means. The British Psychological Society (2 ${ }^{\text {nd }}$ edn. $), \mathrm{pp}: 1-8$.

10. The Sustainable Development Agenda (2019) United Nations Sustainable Development. United Nations.

11. Hannah Dellabella (2017) Top 10 Mental Health Apps. Psychiatry Advisor, New York.

12.Von Schirnding Y, Mulholland C (2017) Health and sustainable development: key health trends. World Health Organization PP: 1-15.

13. (2014) Mental health: A state of well-being. World Health Organization. 
(C) (P) This work is licensed under Creative

DOI: 10.32474 /SJPBS.2020.04.000184

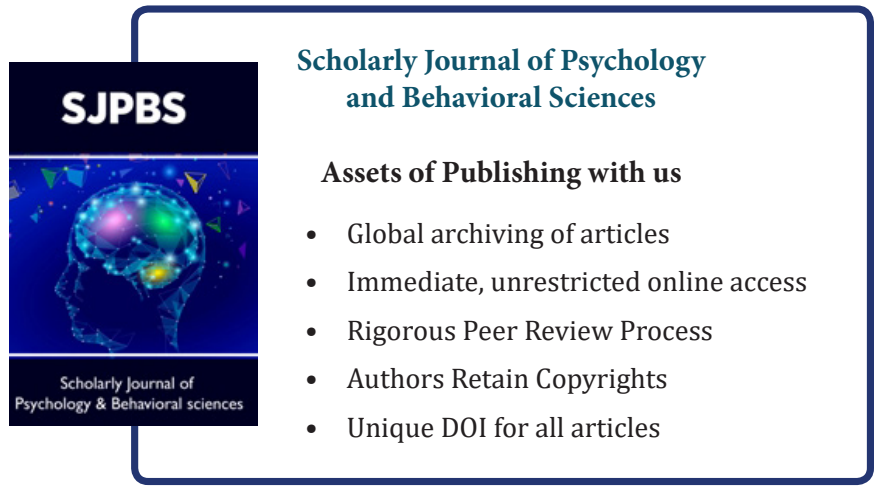

